\title{
Exploring the Possibility of Virtual Reality Exergaming as a Cognitive Screening System
}

\author{
Alex Shaw \\ School of Computer Science \\ The University of Auckland \\ $\underline{\text { l.shaw@ auckland.ac.nz }}$
}

\author{
Jude Buckley \\ School of Psychology \\ The University of Auckland \\ jb.h.adv@xtra.co.nz
}

\author{
Paul Corballis \\ School of Psychology \\ The University of Auckland \\ p.corballis@auckland.ac.nz
}

\author{
Christof Lutteroth \\ Department of Computer Science \\ The University of Bath \\ c.lutteroth@bath.ac.uk
}

\author{
Burkhard C. Wünsche \\ School of Computer Science \\ The University of Auckland \\ b.wuensche@auckland.ac.nz
}

\begin{abstract}
Regular screening is important for early detection of cognitive impairment. Standard cognitive screening tests can be time consuming and tedious. Individuals at risk of cognitive decline should be doing regular exercise, and Virtual Reality (VR) exergames are one way to motivate them to do so. If these exergames can include non-obtrusive cognitive tests, then users can be regularly screened without it being such a tedious process. However, exergaming and VR might threaten the validity of these tests. This paper investigates whether this is likely to be the case and discusses some practical concerns around this approach of screening for cognitive decline. The results of this study suggest that standard cognitive tests are not rendered invalid in VR exergaming. However, more work is needed to investigate the effects of integrating tests into gameplay elements.
\end{abstract}

\section{Introduction}

Cognitive functions tend to decline with normal ageing $[1,2]$. Decline in executive functions - the set of processes that define cognitive control [3] - is associated with a severe drop in quality of life and an increase in mortality rate [4]. The growing elderly population in many countries means that management of cognitive impairment disorders will place an increasing burden on societal resources [5].

Treatment and management of these disorders is most effective when they are identified early [6]. In many cases there is a long initial period where deterioration is present but not obvious [7], resulting in under-diagnosis of impairment [8,9]. Regular screening of executive function can help to catch these cognitive impairments early. However, there are several practical issues that impede early detection methods. Traditional methods of testing for cognitive impairment are time-consuming, costly, and tedious for individuals to complete. When done in a clinic or lab, it costs time from medical staff. When done outside of a clinic, the tedium makes adherence unlikely.

Regular exercise is another case where adherence to medically prescribed independent programs is low [10]. This is unfortunate, since regular exercise is one effective way to prevent, delay, or mitigate age related cognitive decline [11], and it offers many other health benefits.

One tool for increasing exercise program adherence that has shown promising results in past research is exergames, or exercise video games [12]. Exergames aim to increase motivation to exercise by making it more enjoyable and directing motivation to play video games into exercise.

An interesting characteristic of exergames is that they have the potential to make exercise occur in a fairly controlled scenario. While the user's attention is focused on the game display, the exergame has control over what they will be seeing. The rules of the exergame can regulate the intensity of the exercise [13]. This control makes exergames a potential candidate as a vehicle for cognitive tests. If the user regularly plays an exergame as part of an exercise regimen, the integration of cognitive tests into said exergame would enable regular cognitive screening, and without the requirement of active involvement from medical staff. If the tests can be integrated into the actual gameplay of the exergame in a non-obtrusive fashion, then the tedium of regularly doing cognitive tests can be minimised. If the user's performance in game-based tests decreases or exhibits unexpected characteristics, then the user can be flagged for full cognitive assessment and diagnosis. 
Immersive VR offers some benefits in exergames, showing potential to increase motivation to play by increasing the sense of presence in the game [14], and to increase total exercise quantities by dissociating the user from the unpleasant aspects of exercise such as fatigue. It also offers some potential for exergaming as a platform for cognitive testing, as an immersive experience allows for much greater control of the environment the user is exposed to.

However, using exergaming and VR as a tool for cognitive testing comes with several concerns. Standard cognitive tests have not been designed with this use case in mind. The fact that exercise influences cognitive performance is well established. It is possible that performing a test while playing an exergame or while immersed in a virtual environment will also affect performance in such a way as to influence the validity of the test, and thus its potential as a screening tool.

This paper describes the implementation of a standard cognitive test, the AX-Continuous Performance Task (AXCPT) within a VR exergame, and conducts a study to determine whether the introduction of exergaming or VR changes test performance. This paper is focused on whether the introduction of these elements influences the test, but leaves the integration of such a test into the gameplay elements themselves for future work.

The primary contribution of this paper is evidence supporting the validity of a standard cognitive test, the AXCPT, when implemented in a VR exergame. This paper offers insight into how exercise and exergaming influence performance in such a test.

\section{Related Work}

\subsection{Cognitive Assessment}

Past work has proposed screening with digital tools or serious games as a substitute for traditional methods. In addition to digital methods, several papers also identify VR as a potentially useful tool, though unlike this work, they mostly focus on non-immersive VR. However, the potential impact of the introduction of VR to testing methods has not yet been investigated.

Several works, such as those of Zuchella et al. [15] and Valladares-Rodríguez et al. [16] identify the advantages of such an approach both in terms of time and cost saving, and in terms of improving ecological validity of cognitive tests. We believe that immersive VR offers the same ecological validity benefits, possibly to a greater degree.

Negut et al. [17] conducted a meta-analysis of literature on the use of VR technology in neuropsychological analysis. Their results support the theory that VR based measures are suitable for the detection of cognitive impairment. They suggest that VR measures may be suitable both for research and clinical practice. They also identify the need for neuropsychological tests that assess the subject in an environment closer to real life than the laboratory.

Pugnetti et al. [18] also look at the role of VR in analysing and treating cognitive impairments such as age-related cognitive impairment. In particular, they identify VR as a useful tool for solving the problem of transfer effects. They also identify evaluation of higher cognitive functions as an area sorely lacking technological aids for rehabilitation, and suspect that VR may be superior to traditional digital procedures.

Tong et al. [19] evaluate some simple, purpose-built serious games for cognitive assessment. They use standard tests to get a baseline for the participants' cognitive abilities, then assessed how performance in the games correlated to test performance. They found game performance had a significant correlation with all cognitive abilities measured with standard tests.

Overall, these works come to the same conclusions: it is important to screen for cognitive impairment, that virtual tools are likely to be effective for this purpose, and that they may be very useful for providing test tasks that more closely approximate real life ones.

In an example of testing replicating real life scenarios, Seo et al. [20] used movement analysis in a virtual environment to screen for mild cognitive impairment. In their work, participants were required to complete tasks in VR that mimicked real-world scenarios: withdrawing money from an ATM and catching a bus. They found that analysing speed and movement trajectory was an effective means of identifying subjects suffering from cognitive impairment.

\subsection{Cognitive Control}

One test frequently used for assessment of cognitive function is the AX Continuous Performance Task (AXCPT). The AXCPT assesses proactive and reactive cognitive control. The relationship cognitive control holds with factors such as video game play and exercise has been investigated in several past works.

Time spent playing interactive video games has been indicated to have an influence on proactive, but not reactive cognitive control. Bailey et al. [21] evaluated the performance of individuals with high and low experience playing video games in a Stroop task. This study evaluated the cognitive control levels of the gamers outside of the context of a game environment. 
This is of particular interest to us, as individuals who play video games are one major target demographic for exergames. It is also a possibility that when performing a task to measure cognitive control while playing a game, performance is more likely to match the profile of an individual who plays video games.

The effects of exercise on cognitive control have been well researched. Davranche and McMorris evaluated performance in a Simon task while cycling [22]. Reaction times improved when completing the task simultaneously with exercise, but inhibitory control decreased. This is similar to the results of studies on working memory, where exercise appears to offer a benefit in response times, but reduce task accuracy [23]. The suggested mechanism for this effect, exercise increasing the concentration of catecholamines in the brain resulting in faster processing is something that would likely have similar results during a cognitive control task.

In contrast to the above study, in an evaluation of children, Hillman et al. [24] found that the addition of moderate intensity exercise improved inhibitory control in a modified Flanker task. Unlike our work and the above study, this study conducted the cognitive test following the exercise, rather than simultaneously with it.

The concept of a VRAXCPT has been explored before. Rizzo et al. designed a VR classroom environment aimed at children with Attention Deficit Disorder that encompasses a number of learning tools and cognitive tests [25]. Among the tests in their classroom is a version of the AXCPT. They also identify virtual environments as useful tools not just for assessing cognitive function but also for treating cognitive impairments, such as age related cognitive impairment.

In summation, the literature shows a strong grounding for the use of serious games and potentially exergames to perform cognitive screening. Little work has been done using immersive VR or exergames however, and there is only indirect evidence for how these factors may affect test performance.

\section{Design}

In order to investigate whether the introduction of immersive VR and exergaming invalidates cognitive tests, we present a VR version of the AXCPT, integrated into an exergame.

The AXCPT was chosen due to the fact that it measures cognitive control, which is influenced by age $[26,27]$. The requirement for constant attentional focus while performing an AXCPT test poses an interesting interaction with the requirement for shifting focus while playing an exergame.

We built upon an existing exergame that utilises immersive VR, presented in work by Shaw et al. [28]. This exergame is well suited for a target audience due to the visually simple environment and straightforward navigation within the environment, minimising cognitive overhead.

This exergame is played using a computer connected exercycle. As the player cycles, they move along a linear track in accordance with how fast they are cycling. Throughout the track are a variety of obstacles the player must avoid, and rewards the player is encouraged to collect, visible in Figure 1. The player earns points by successfully avoiding obstacles and collecting certain varieties of reward, and loses points when they fail to avoid an obstacle.

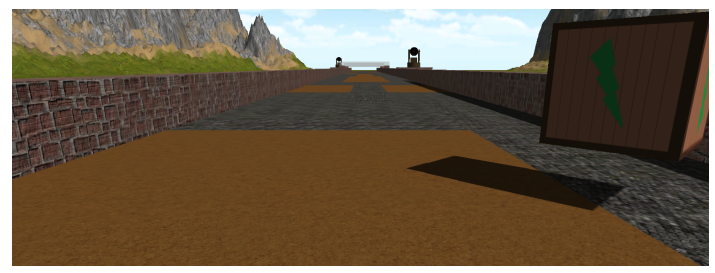

Figure 1. Screenshot from the exergame used in our study.

The player's head and upper body are tracked using a depth camera mounted in front of the exercycle. This detects motions such as leaning from side to side and ducking, which the player does in order to steer and avoid obstacles.

The game is presented to the player using a head-mounted-display to achieve an immersive VR experience. During our study, the Oculus Rift DK2 headset was used.

\subsection{AX-Continuous Performance Task (AXCPT)}

The AXCPT is a cognitive test that measures an individual's sustained cognitive control. The AXCPT is a popular test for examining the proactive and reactive control of the subject. Proactive control is the anticipation and prevention of interference before it occurs, and reactive control is detection and resolution of interference after it occurs. Successful and effective cognition appears to depend on a combination of the two. Cognition shifts more towards reactive control over proactive as adults age [26, 27].

In the AXCPT test, subjects are presented with a series of alternating cues and probes. Normally, these alternate between the cue letter ' $A$ ', and the probe letter 
' $\mathrm{X}$ '. At each letter, the subject must make either a target or non-target response, typically by pressing different buttons or letters on a keyboard. When the subject sees an ' $\mathrm{X}$ ' probe that was immediately preceded by an ' $\mathrm{A}$ ' cue, they are to give the target response. When they see anything else (including the 'A' cues), they are to give the non-target response. Cues and probes are presented with a short delay between them, in which a neutral symbol such as ' + ' is displayed. The vast majority of cue-probe pairs follow the 'A', ' $\mathrm{X}$ ' pattern, establishing a tendency in the subject to give the target response upon seeing an ' $\mathrm{X}$ ' cue. The sequence of ' $\mathrm{A}$ ' followed by ' $\mathrm{X}$ ' is an ' $\mathrm{AX}$ ' prompt.

In addition to 'AX' prompts, a small portion of the cue-probe pairs do not follow this pattern. In some cases, there will be an alternate letter in place of the 'A', but the following letter will still be an ' $X$ '. This pattern is a ' $\mathrm{BX}$ ' prompt. In other cases, the ' $\mathrm{A}$ ' cue is followed by a letter that is not ' $\mathrm{X}$ '. This pattern is an 'AY' prompt. Finally, in some cases both letters may not fit the pattern, giving a 'BY' prompt. As the subject is predisposed to the pattern of alternating between non-target and target responses, responding appropriately to $\mathrm{AY}, \mathrm{BX}$, or BY prompts requires the subject to exercise cognitive control.

Good proactive control increases accuracy when responding to $\mathrm{BX}$ prompts as the subject is able to use the initial cue of the ' $\mathrm{B}$ ' cue to inhibit the positive response upon seeing the ' $\mathrm{X}$ ' probe. However, it can reduce performance when responding to AY prompts, because in this case the expectancy generated by the initial cue is invalid [29]. In contrast, reactive control increases performance on AY prompts as the subject is able to react appropriately after the interference of the invalid probe has occurred.

\subsection{Virtual Reality AX Continuous Performance Task (VRAXCPT)}

In the VRAXCPT, the task prompts are presented floating in 3D space in front of the user. If the user is looking forward (down the track), the prompts will appear approximately in the centre of the user's field of view. The linear nature of the game environment is a major advantage here, as the user is thus always aware of the direction they have to be looking to see the cues, both in terms of their bodily orientation, and also the orientation of their virtual view relative to the environment.

Traditionally, the AXCPT is presented with prompts appearing against a plain, single colour background. This presents a problem when translating the test to $\mathrm{VR}$, as the presence of a virtual environment could be considered a defining characteristic of a VR experience. If there is no environment, the subject is simply performing a traditional AXCPT with a weighted attachment on their face. Thus to compare the VRAXCPT with the traditional AXCPT, it is necessary to have an environment, and accept that a differing level of visual complexity between the tests is simply one of the factors compared between the two.

The virtual environment in the exergame we use is visually neutral and simple, as can be seen in Figure 2, so the increase in mental load from the surroundings should be minimised. In a similar fashion, adding exercise lends itself to a moving environment, but the nature of the environment we use stays constant as the user moves through it, minimising the impact of this factor.

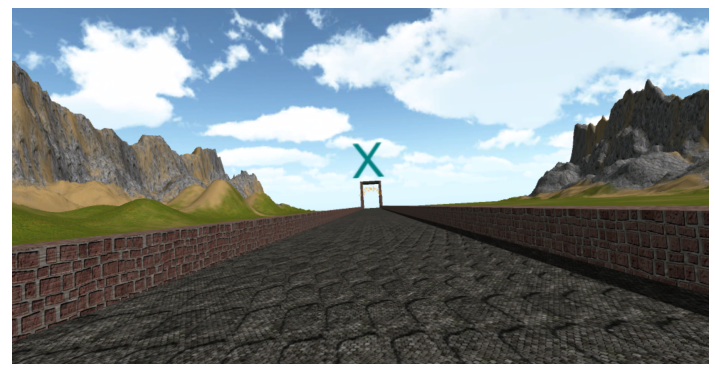

Figure 2. ' $\mathrm{X}$ ' cue shown in the virtual environment.

\section{User Study}

A cross-sectional within-subjects study was conducted to determine the validity of a VR exergame implementation of the AXCPT, and to see the effects of exercise and increased cognitive load via the addition of simultaneous gameplay tasks on the user's test performance.

Our study contained four test conditions:

1. Traditional: Performed on a regular computer screen, using the keyboard.

2. VR: Performed in a stationary VR environment, using the keyboard.

3. VR + Exercise: Performed in a VR environment linked to movement on the exercycle, using triggers attached to the exercycle.

4. VR Exergame: Performed in a VR exergame based on the exercycle, using triggers attached to the exercycle.

Each test condition contained two blocks of 50 cue-probe pairs each, with each block separated by a 30 second break. In total, each condition had 100 pairs. 
The traditional condition was completed using the E-Prime AXCPT test software. In this condition test prompts appeared on screen against a plain black background, alternating between white text for the cues, and teal text for the probes.

In the basic VRAXCPT condition, the gameplay and physical control components of the exergame are disabled, such that the user is stationary in the VR environment. The VR environment is the same environment as in the exercise and gaming conditions, providing the same level of background visual complexity. They are still able to look around, but not to move, and there are no required gameplay tasks. In order to maintain visual contrast between the test prompts and the environment, in the VR versions of the test, the prompts alternate between red and teal.

In the VR + exercise condition, the gameplay components are disabled, but the user moves through the environment according to their cycling speed.

In the VR Exergame condition, the gameplay elements are all enabled, forcing the user to split their focus between the AXCPT and the game's tasks.

The AXCPT and VRAXCPT tasks were divided such that $70 \%$ of the prompts were target (A-X) prompts. The remaining $30 \%$ of prompts were divided evenly between the 3 non-target prompt types. $10 \%$ were an $\mathrm{A}$ followed by something other than an $\mathrm{X}$ (correct cue, incorrect probe), $10 \%$ were a something other than an A followed by an X (incorrect cue, correct probe), and the last $10 \%$ were something other than an A followed by something other than an X (incorrect cue and probe). This is consistent with the task structure used by Marcora et al. [30], which was intended to be mentally intensive, which is important for determining if the test will remain valid under these constraints.

In all conditions, the window in which the cue or probe is displayed and the participant's response counted lasted for 1 second. Failure to respond within this window was recorded as no response. The delay time between the cue and probe, and between the probe and the cue of the next pair was randomised between 1 and 1.5 seconds to avoid a fixed rhythm.

A total of 23 individuals participated in the study, 17 male and 6 female, all of which were able to complete it. Participants were young adults with a median age of 21 , to minimise the possibility of undiagnosed dementia disorders interfering with results. Informed consent was obtained from all participants, and the study was approved by the University of Auckland Ethics Committee (reference number: 016925).

Participants completed a pre-test questionnaire to provide general demographic data such as age and gender, and baseline self-report measures of the typical number of hours spent exercising and playing video games each week. Participants were then given a written outline of the test procedure and conditions, as well as instructions on the AXCPT procedure and on how to play the exergame.

Prior to the test conditions, the participants completed a two minute practice block of the standard AXCPT. They then completed a two minute practice session of the exergame, with no AXCPT test elements present. These practice sessions removed the potential issue of participants starting with the simultaneous VRAXCPT Exergame condition and having to learn how to perform both tasks simultaneously.

The participants then completed the four test conditions in a counterbalanced order determined by the method of Latin Squares. Participants were given a 2-3 minute break between each of the conditions.

\subsection{Measures}

Error Rate: The number of times a participant responds incorrectly to a cue or a probe. There are four main types of error based on the particular arrangement of cue and probe: AX, AY, BX, and BY. Each of these can be further divided by whether an error occurred in responding to the cue or the probe, but we primarily deal with errors occurring in the trigger response. Errors on $\mathrm{BX}$ cues correspond to failures of proactive cognitive control, while errors on AY cues correspond to failures of reactive cognitive control.

Response Rate: The frequency at which participants respond or fail to respond within the 1 second window.

Reaction Time: The average time in seconds for participants to react to the cue, and the average time in seconds for the participants to react to the probe.

Focus Distribution: In the three VR conditions, the amount of time spent looking at the cues, and the amount of time spent looking at the environment or gameplay elements. This was calculated based on the divergence of the forward vector of the virtual camera in the 3D environment from the vector required to centre the AXCPT prompts in the participant's field of view. Measurements of this vector were taken every 0.5 seconds during each of the VR conditions.

\section{Results}

The normality and sphericity assumptions of RM-ANOVA were tested with the Shapiro-Wilk test and Mauchly's sphericity test respectively.

Pearson correlation analyses were used to examine the association between the participant information gathered pre-test, and the various measures. 
Table 1. Summary of mean participant responses (per 100 tests) and reaction times (seconds)

\begin{tabular}{l|rrrr}
\hline & Traditional & VR & VR + Exercise & VR Exergame \\
\hline Cue, no response & 0.30 & 0.57 & 0.91 & 8.83 \\
Cue, correct response & 99.43 & 99.34 & 98.96 & 90.65 \\
Cue, incorrect response & 0.26 & 0.09 & 0.13 & 0.52 \\
Probe, no response & 0.39 & 0.48 & 1.13 & 7.09 \\
Probe, correct response & 93.74 & 94.30 & 94.61 & 83.57 \\
Probe, incorrect response & 5.87 & 5.22 & 4.26 & 9.34 \\
Cue reaction time & 0.318 & 0.378 & 0.494 & 0.570 \\
Probe reaction time & 0.352 & 0.391 & 0.500 & 0.567 \\
\hline
\end{tabular}

Table 2. Incorrect probe response error rates (per 100 tests)

\begin{tabular}{l|rrrr}
\hline & Traditional & VR & VR + Exercise & VR Exergame \\
\hline AX & 3.91 & 3.11 & 2.67 & 6.71 \\
AY & 22.17 & 20.0 & 10.0 & 13.04 \\
BX & 6.96 & 8.70 & 12.61 & 26.96 \\
BY & 2.17 & 1.74 & 1.3 & 6.52 \\
\hline
\end{tabular}

Test performance is comparable across all conditions but the VR Exergame condition. In general, performance in the traditional AXCPT is marginally higher than the VR conditions, but still relatively close. However, performance in the VR exergame condition is significantly worse than in the other conditions. In particular, the rate of participants failing to respond to a cue or a probe is substantially higher.

Reaction times gradually increase with the complexity of the task, with the regular AXCPT having the lowest times, and the VR Exergame having the highest. Reaction times are quite consistent between cue and probe cues for all four conditions. The differences in reaction times between all conditions are significant $(\mathrm{p}<0.001)$, for both cue and probe reactions.

As discussed in the design of the AXCPT, the visual complexity of the task surroundings increases slightly going from the traditional test to the VR version, and again going from VR to VR + exercise. This could be responsible for some of the increase in reaction time and rates of no response. This may account for the contrast with the findings of Davranche and McMorris, who found reaction times during a Simon task were faster when the task was performed simultaneously with exercise [22]. In their study, there was no use of VR, and thus no virtual environment. As our results show an increase in reaction times both with and without exercise, the increasing complexity of the virtual environment is a plausible explanation.

Table 2 shows the mean rate of each type of error per participant (100 tests). Note that these values only include cases where the participant gave an incorrect response to the probe part of the test, as incorrect responses to the cue should be unrelated to cognitive control and likely arise from genuine mistakes or distraction. Cases where the participant gave an incorrect response to the cue but still gave a correct response to the probe, or cases where they gave no response are not included. The two exercise conditions have considerably more $\mathrm{BX}$ errors as a percentage of overall errors. The Exergame condition has a much higher percentage of AX errors than the other conditions, in particular the other VR conditions.

\section{Discussion}

Interestingly, in the two conditions where exercise was present, there was a higher rate of BX errors than of AY errors. The rate of AY errors was significantly lower in the two exercise conditions than in the two conditions without exercise. Thus our results suggest that exercise increases the use of reactive cognitive control strategies, but decreases proactive control. This is consistent with the idea that as individuals become tired, they shift toward the more computationally efficient reactive cognitive control [29]. In particular, the VR exergame condition has a very high rate of $\mathrm{BX}$ errors. This may be because the increased cognitive load added by the game makes it more difficult for the participant to keep track of the initial cue and thus they just react to probes as they appear.

Increased cognitive load leads to mental fatigue which reduces exercise performance [30]. Unsurprisingly, $75 \%$ of participants rated the exergame condition as the most mentally tiring of the four conditions, and only a single participant rated it as the least tiring. In contrast, none of the participants rated the VR + exercise condition as the most tiring, and $30 \%$ 
rated it as the least tiring. Despite this, our results show significantly higher exercise performance during the exergame condition than in the $\mathrm{VR}+$ Exercise condition $(\mathrm{p}<0.01)$. Mean distance travelled in the $\mathrm{VR}+$ exercise condition was $2.87 \mathrm{~km}$, while mean distance travelled in the exergame condition was $3.25 \mathrm{~km}$. It is likely that any reduction in exercise performance due to mental fatigue was counteracted by the motivational effects of the game. It is also possible that any reduction in exercise performance due to mental fatigue would not show up in the relatively short duration of the experimental task.

The shift towards reactive strategies in the exercise conditions may hold some implications for the design of exergames. If the player is more likely to simply react rather than prepare when playing an exergame, then it is sensible to design gameplay elements that can be handled reactively, otherwise the player may feel that the game is unfair as they are presented with elements they are unable to handle. It is also possible that as the player continues to exercise and grows more tired, they will shift further towards a reactive approach. This could be handled by adjusting the distribution of gameplay elements through the level: if there are gameplay elements that require a proactive approach then their density might be high at the start of play and decrease over time, being replaced by more elements that can be handled reactively.

In the case of the gameplay elements present in this exergame, all are able to be handled using either proactive or reactive control. All obstacles and bonuses can be seen ahead of time, and the cannons fire at predictable intervals, enabling a proactive approach. The time between an obstacle becoming immediately apparent and the player interacting with it is sufficiently long for it to be handled reactively.

Although the error and non-response rates in the exergame condition are much higher, the ordering of frequency of error types in the exergame condition is the same as that of the $\mathrm{VR}+$ Exercise condition. The exergame condition has a higher proportion of $\mathrm{AX}$ and BY errors, presumably because interference from game elements is just as likely to occur with any cue/probe combination, and thus skews error rates towards the distribution of probe types.

Table 3 shows a selection of correlations between measures from the demographic questionnaire, and successful responses to test probes. In contrast to the findings of Bailey et al. [21], in no condition do our results show a significant correlation with time spent playing video games and either AY or BX error rates, and thus proactive or reactive control. Though time spent playing video games did have a significant moderate positive correlation with failures to respond to
AX prompts in the VR condition, this is only barely significant $(\mathrm{p}=.049)$ and we believe it likely to be coincidence. This aligns our results with the suggestion that prior studies which showed a difference between gamers and non gamers were flawed, and is consistent with other research that shows either no significant difference or only limited differences between the two populations $[31,32]$.

Time spent in regular exercise had a significant moderate positive correlation with incorrect AY responses in the VR condition, and with no response to $\mathrm{AX}$ and $\mathrm{BY}$ prompts in the VR exergame condition. This is surprising, although we would expect to see less of a shift from proactive to reactive control with increased physical fitness, the fact that there is a deterioration in reactive performance with no associated improvement in proactive performance is unexpected.

We marked an angle of greater than $30^{\circ}$ between the participant's view vector and the vector to the test cue as an indication that the participant's focus was not on the cue at that point in time. The field of view of the Oculus Rift DK2 is $100^{\circ}$ which means a $30^{\circ}$ divergence places the cue well away from the centre of the participant's view. Table 4 shows a summary of participant view orientation. Participant focus was primarily directed at the AXCPT cues in the VR and VR + Exercise conditions. In the VR Exergaming condition, focus was frequently diverted towards gameplay elements.

Participant focus is a likely cause of the high levels of AX and "no response" errors in the Exergame condition. It is reasonable to assume that errors due to participants' focus being on gameplay elements are distributed proportionally across the different test types. Thus, due to $70 \%$ of the tests being AX, we would expect to see $\mathrm{AX}$ errors represented at a rate closer to $70 \%$ than in other conditions. A greater overall error rate unrelated to cognitive control leads to a distribution of errors closer to the distribution of test types.

\subsection{Limitations}

This study analyses test performance across the whole group of participants. Although it does find group performance is largely comparable between the conditions, an ideal assessment of comparability would be to see if rank order is preserved. Unfortunately, this study design means that small differences in performance can result in large differences in rank.

100 total cue-probe pairs means that each error type is encountered 10 times per participant. With successful responses being generally high across the board, the difference between successfully responding to 7 pairs, and successfully responding to 8 pairs causes 
Table 3. Pearson correlations of time spent playing videogames and exercising, perceived fitness, self reports of physical and mental tiredness with frequency of correct responses to test probes.

\begin{tabular}{l|rrrrr}
\hline & Exercise Time & Game Time & Fitness & Phys. Tired & Ment. Tired \\
\hline Screen AX & -0.033 & -0.051 & 0.110 & $0.469^{\mathrm{a}}$ & -0.099 \\
VR AX & -0.344 & 0.023 & -0.307 & -0.097 & -0.367 \\
EX AX & 0.085 & -0.106 & 0.056 & 0.053 & 0.032 \\
EG AX & $-0.512^{\mathrm{a}}$ & -0.185 & -0.122 & 0.222 & 0.085 \\
Screen AY & -0.233 & 0.197 & -0.334 & $0.491^{\mathrm{a}}$ & 0.024 \\
VR AY & $-0.637^{\mathrm{a}}$ & -0.036 & -0.298 & 0.397 & 0.295 \\
EX AY & -0.186 & 0.122 & -0.075 & 0.180 & 0.117 \\
EG AY & -0.063 & -0.106 & 0.036 & 0.279 & 0.192 \\
Screen BX & -0.288 & 0.145 & -0.319 & -0.085 & -0.182 \\
VR BX & -0.163 & 0.129 & -0.177 & -0.291 & -0.311 \\
EX BX & -0.162 & 0.039 & -0.156 & -0.019 & -0.152 \\
EG BX & -0.374 & -0.028 & -0.259 & 0.063 & 0.213 \\
Screen BY & -0.148 & 0.307 & -0.245 & 0.141 & -0.091 \\
VR BY & -0.265 & 0.026 & -0.290 & -0.215 & -0.128 \\
EX BY & 0.117 & 0.236 & -0.026 & 0.235 & 0.023 \\
EG BY & $-0.476^{\mathrm{a}}$ & 0.166 & -0.423 & -0.058 & 0.197 \\
\hline
\end{tabular}

Notes: $\mathrm{N}=23$. Correlations significant at the $\mathrm{p}<0.05$ level are denoted by ${ }^{\mathrm{a}}$.

Table 4. Summary of view orientation in VR conditions

\begin{tabular}{l|rrr}
\hline & VR & VR + Exercise & VR Exergame \\
\hline Mean variation (degrees) & 13.2 & 13.5 & 17.1 \\
$\%$ time not focused on prompt & 2.3 & 3.0 & 7.3 \\
\hline
\end{tabular}

a substantial difference in ranking.

While rank order appears roughly comparable (participants who do well in one condition tend to do well in others, and vice versa), such a measure is not accurate enough in this study to draw conclusions.

Measurements of reaction times are only present for occasions where the participant reacted during their window of opportunity. In cases where a participant reacted slowly enough that they missed the window (counting as no response), this slow reaction was not recorded. In the VR Exergame condition, which had a comparatively high rate of no response, the actual mean reaction time may be slightly higher than reported.

It would be interesting to compare participants' visual focus in the Traditional condition with their focus in the other conditions. Unfortunately our method of tracking their focus relied on the VR headset, and thus was not available in the Traditional condition. Our focus tracking method also assumes that the forward vector of the headset corresponds to where the participant is looking. It is possible that in some cases, the participants' eyes may not have been looking where their head was aimed. However, due to the limited field of view of the headset and normal coordination between head direction and focus [33], this is unlikely. Finally, we chose a conservative value for the field of view cut-off, and thus time spent focused on the cues may be greater in all conditions than we report.

\section{Other Concerns}

The equivalence of test performance in a traditional format and in a VR exergame is not the only concern around the screening approach proposed in this paper.

One major concern with having subjects undergo such regular screening is training effects, which occur in both cognitive exercises and video games. If they are performing the cognitive exercise every few days, will they develop skill at it such that it loses its ability to identify a cause for concern?

Interestingly, the presence of training effects is itself useful information about the likelihood of some form of cognitive impairment. There is evidence to suggest a relationship between training effects or the lack thereof, and cognitive degradation [34, 35]. Some tests are also less susceptible to training effects than others. The AXCPT in particular has shown resistance to training effects [36].

VR exergaming also has some associated practical considerations. Cybersickness, physical discomfort from a headset, and the requirement of a powerful computer are all obstacles towards achieving this goal. 
However, improvements to VR technology mean that these issues are gradually becoming less problematic.

This approach to screening relies on the exergame being able to motivate the user to regularly engage with it. If there is no ongoing data, there is no way to identify a change in performance. As such, any game designed for this purpose faces a demanding requirement: the gameplay must be engaging while still being effective for cognitive testing.

\section{Conclusions}

This study indicates that a VR version of the AXCPT is viable as a tool for cognitive assessment. Performance in the VR version of the test is comparable to performance in the traditional version. Although the presence of VR appears to degrade reaction times in the test, it does not appear to influence cognitive control. As such, we show that the AXCPT is a valid and useful tool for evaluating cognitive control in a VR environment.

Introducing exercise and exergaming does alter performance on the AXCPT, so while the test remains a useful tool in these scenarios, it is important to be aware that any analysis of results obtained in these cases must take into account a shift toward reactive control from the exercise, and a skewing toward the natural distribution of test cues when exergaming.

VR exergaming is a demanding cognitive task that has a substantial influence on the subject's ability to perform a cognitive test. When playing the exergame, the user's attention is divided between the game and the test. The bodily motion controls of the exergame also serve to pull the user's view away from test elements, increasing the likelihood of the user being unable to respond to a test cue in time, even when the test cues are positioned in such a manner as to minimise the effort of returning attention to them after having it diverted.

In the absence of other items requiring cognitive attention, user focus in VR remains on the test elements. The presence of VR does not appear to significantly distract the user from the test, allowing these tests to be carried out without concern that a VR environment will consume too much of the user's attention, though further testing would be required to be certain that this holds if the environment is visually complex.

We do not propose this method of screening as a complete diagnostic tool, but by getting at risk individuals to undergo regular testing, warning signs can be caught early, and individuals flagged for follow-up assessment with a medical professional.

\subsection{Future Work}

There are many cognitive tests besides the AXCPT that are used as assessment tools. To be confident that the results in this paper generalise, these other tests should also be investigated.

As this work currently stands, the AXCPT exists within the VR exergame, but it is not integrated into the gameplay. To perform the test while playing the game requires the user to focus on two demanding tasks at once, with a substantial decrease in their performance as shown by the results here. The next step is to integrate the test into gameplay elements directly, such that to play the game is to perform the test.

An alternative future approach might be to create new cognitive tests directly in the form of gameplay elements, and validate these tests against existing tests for the associated cognitive function.

If it is possible to integrate tests into gameplay elements, then it is possible that some commercial video games have by chance incorporated gameplay elements that would serve as effective tests. A worthwhile future investigation would be to examine the gameplay existing video games to identify cases where these games could be used for cognitive screening purposes.

\section{References}

[1] T. Hedden and J. D. Gabrieli, "Insights into the ageing mind: a view from cognitive neuroscience," Nature reviews neuroscience, vol. 5, no. 2, p. 87, 2004.

[2] D. L. Murman, "The impact of age on cognition," in Seminars in hearing, vol. 36, pp. 111-121, Thieme Medical Publishers, 2015.

[3] A. Miyake, N. P. Friedman, M. J. Emerson, A. H. Witzki, A. Howerter, and T. D. Wager, "The unity and diversity of executive functions and their contributions to complex frontal lobe tasks: A latent variable analysis," Cognitive psychology, vol. 41, no. 1, pp. 49-100, 2000.

[4] J. K. Johnson, L.-Y. Lui, and K. Yaffe, "Executive function, more than global cognition, predicts functional decline and mortality in elderly women," The Journals of Gerontology Series A: Biological Sciences and Medical Sciences, vol. 62, no. 10, pp. 1134-1141, 2007.

[5] T.-T. Dang, P. Antolin, and H. Oxley, "Fiscal implication of ageing: projections of age-related spending," Organisation for Economic Co-operation and Development (OECD) Working Paper No. 305., 2001.

[6] D. L. Weimer and M. A. Sager, "Early identification and treatment of alzheimer's disease: social and fiscal outcomes," Alzheimer's \& Dementia, vol. 5, no. 3, pp. 215-226, 2009.

[7] S. T. DeKosky and K. Marek, "Looking backward to move forward: early detection of neurodegenerative disorders," Science, vol. 302, no. 5646, pp. 830-834, 2003.

[8] S. Lithgow, G. A. Jackson, and D. Browne, "Estimating the prevalence of dementia: cognitive screening in 
glasgow nursing homes," International journal of geriatric psychiatry, vol. 27, no. 8, pp. 785-791, 2012.

[9] H. Magsi and T. Malloy, "Underrecognition of cognitive impairment in assisted living facilities," Journal of the American Geriatrics Society, vol. 53, no. 2, pp. 295-298, 2005.

[10] F. Jones, P. Harris, H. Waller, and A. Coggins, "Adherence to an exercise prescription scheme: the role of expectations, self-efficacy, stage of change and psychological well-being," British journal of health psychology, vol. 10, no. 3, pp. 359-378, 2005.

[11] M. Hirsch and B. Farley, "Exercise and neuroplasticity in persons living with Parkinson's disease.," European journal of physical and rehabilitation medicine, vol. 45, no. 2, pp. 215-229, 2009.

[12] D. E. Warburton, S. S. Bredin, L. T. Horita, D. Zbogar, J. M. Scott, B. T. Esch, and R. E. Rhodes, "The health benefits of interactive video game exercise," Applied Physiology, Nutrition, and Metabolism, vol. 32, no. 4, pp. 655-663, 2007.

[13] A. Keesing, M. Ooi, O. Wu, X. Ye, L. Shaw, and B. C. Wünsche, "Hiit with hits: Using music and gameplay to induce hiit in exergames," in Proceedings of the Australasian Computer Science Week Multiconference, p. 36, ACM, 2019.

[14] R. M. Ryan, C. S. Rigby, and A. Przybylski, "The motivational pull of video games: A self-determination theory approach," Motivation and emotion, vol. 30, no. 4, pp. 344-360, 2006.

[15] C. Zucchella, E. Sinforiani, C. Tassorelli, E. Cavallini, D. Tost-Pardell, S. Grau, S. Pazzi, S. Puricelli, S. Bernini, S. Bottiroli, et al., "Serious games for screening pre-dementia conditions: from virtuality to reality? a pilot project," Functional neurology, vol. 29, no. 3, p. $153,2014$.

[16] S. Valladares-Rodríguez, R. Pérez-Rodríguez, L. Anido-Rifón, and M. Fernández-Iglesias, "Trends on the application of serious games to neuropsychological evaluation: a scoping review," Journal of biomedical informatics, vol. 64, pp. 296-319, 2016.

[17] A. Negut, S.-A. Matu, F. A. Sava, and D. David, "Virtual reality measures in neuropsychological assessment: a meta-analytic review," The Clinical Neuropsychologist, vol. 30, no. 2, pp. 165-184, 2016.

[18] L. Pugnetti, L. Mendozzi, A. Motta, A. Cattaneo, E. Barbieri, and A. Brancotti, "Evaluation and retraining of adults' cognitive impairments: Which role for virtual reality technology?," Computers in Biology and Medicine, vol. 25, no. 2, pp. 213-227, 1995.

[19] T. Tong, V. Guana, A. Jovanovic, F. Tran, G. Mozafari, M. Chignell, and E. Stroulia, "Rapid deployment and evaluation of mobile serious games: A cognitive assessment case study," Procedia Computer Science, vol. 69, pp. 96-103, 2015.

[20] K. Seo, J.-k. Kim, D. H. Oh, H. Ryu, and H. Choi, "Virtual daily living test to screen for mild cognitive impairment using kinematic movement analysis," PloS one, vol. 12, no. 7, p. e0181883, 2017.

[21] K. Bailey, R. West, and C. A. Anderson, "A negative association between video game experience and proactive cognitive control," Psychophysiology, vol. 47, no. 1, pp. 34-42, 2010.

[22] K. Davranche and T. McMorris, "Specific effects of acute moderate exercise on cognitive control," Brain and cognition, vol. 69, no. 3, pp. 565-570, 2009.
[23] T. McMorris, J. Sproule, A. Turner, and B. J. Hale, "Acute, intermediate intensity exercise, and speed and accuracy in working memory tasks: a meta-analytical comparison of effects," Physiology \& behavior, vol. 102, no. 3-4, pp. 421-428, 2011.

[24] C. H. Hillman, M. B. Pontifex, L. B. Raine, D. M. Castelli, E. E. Hall, and A. F. Kramer, "The effect of acute treadmill walking on cognitive control and academic achievement in preadolescent children," Neuroscience, vol. 159, no. 3, pp. 1044-1054, 2009.

[25] A. A. Rizzo, J. G. Buckwalter, T. Bowerly, C. Van Der Zaag, L. Humphrey, U. Neumann, C. Chua, C. Kyriakakis, A. Van Rooyen, and D. Sisemore, "The virtual classroom: a virtual reality environment for the assessment and rehabilitation of attention deficits," CyberPsychology \& Behavior, vol. 3, no. 3, pp. 483-499, 2000.

[26] T. S. Braver, J. L. Paxton, H. S. Locke, and D. M. Barch, "Flexible neural mechanisms of cognitive control within human prefrontal cortex," Proceedings of the National Academy of Sciences, vol. 106, no. 18, pp. 7351-7356, 2009.

[27] K. Jimura and T. S. Braver, "Age-related shifts in brain activity dynamics during task switching," Cerebral Cortex, vol. 20, no. 6, pp. 1420-1431, 2009.

[28] L. A. Shaw, B. C. Wünsche, C. Lutteroth, S. Marks, J. Buckley, and P. Corballis, "Development and evaluation of an exercycle game using immersive technologies," in 8th Australasian Workshop on Health Informatics and Knowledge Management (HIKM 2015), vol. 164 of CRPIT, pp. 75-85, ACS, 2015.

[29] T. S. Braver, "The variable nature of cognitive control: a dual mechanisms framework," Trends in cognitive sciences, vol. 16, no. 2, pp. 106-113, 2012.

[30] S. M. Marcora, W. Staiano, and V. Manning, "Mental fatigue impairs physical performance in humans," Journal of Applied Physiology, vol. 106, no. 3, pp. 857-864, 2009.

[31] W. R. Boot, A. F. Kramer, D. J. Simons, M. Fabiani, and G. Gratton, "The effects of video game playing on attention, memory, and executive control," Acta psychologica, vol. 129, no. 3, pp. 387-398, 2008.

[32] K. Murphy and A. Spencer, "Playing video games does not make for better visual attention skills.," Journal of Articles in Support of the Null Hypothesis, vol. 6, no. 1, 2009.

[33] M. Gresty, "Coordination of head and eye movements to fixate continuous and intermittent targets," Vision research, vol. 14, no. 6, pp. 395-403, 1974.

[34] D. Darby, P. Maruff, A. Collie, and M. McStephen, "Mild cognitive impairment can be detected by multiple assessments in a single day," Neurology, vol. 59, no. 7, pp. 1042-1046, 2002.

[35] K. Duff, L. J. Beglinger, S. K. Schultz, D. J. Moser, R. J. McCaffrey, R. F. Haase, H. J. Westervelt, D. R. Langbehn, J. S. Paulsen, and H. S. Group, "Practice effects in the prediction of long-term cognitive outcome in three patient samples: A novel prognostic index," Archives of Clinical Neuropsychology, vol. 22, no. 1, pp. 15-24, 2007.

[36] J. M. Halperin, V. Sharma, E. Greenblatt, and S. T. Schwartz, "Assessment of the continuous performance test: Reliability and validity in a nonreferred sample.," Psychological Assessment: A Journal of Consulting and Clinical Psychology, vol. 3, no. 4, p. 603, 1991. 